\title{
Heavy-Elements Reactions DataBase (HERDB): relativistic ab initio geometries and energies for actinide compounds.
}

\author{
Nikolai Andreadi, Artem Mitrofanov, Petr Matveev, Anna Volkova, Stepan Kalmykov \\ Chemistry Department, Moscow State University, 119991, Russia, Moscow, Leninskie Gory 1-3 \\ Corresponding author: nikolayandreadi@gmail.com
}

\section{Uranyl fluoride structure}

Most geometry optimization tasks for the uranyl fluoride leads to the flat conformation (fig. S1a). Though, the presence of a non-flat conformation (fig. S1b) is discussed in some papers. We had to perform manual correction of initial coordinates for the geometry optimization to get the non-flat conformation with a lower energy. It is interesting, that flat structure can be observed in the systems with presence of solvent. The more detailed discussion of $\mathrm{UO}_{2} \mathrm{~F}_{2}$ geometry can be found in a work by Wang and Pitzer ${ }^{1}$.

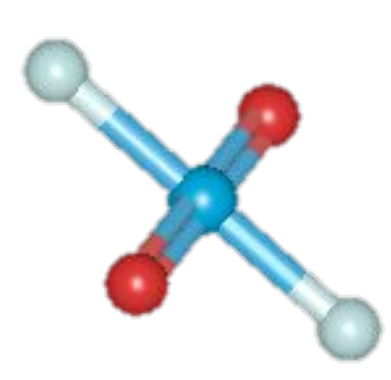

(a) flat conformation (-28300.0255 hartree)

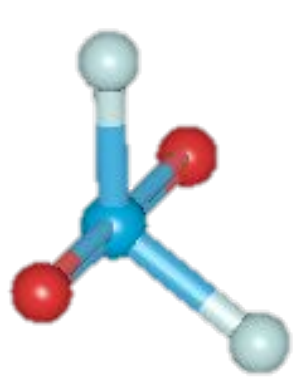

(b) non-flat conformation (-28300.0306 hartree)

Fig. S1 Uranyl fluoride conformations.

\section{Hydroxide structures}

For the uranyl hydroxide and some other hydroxides, one or more hydrogen atoms were deflected from the O-An-O plane. In this paper ${ }^{2}$ it was shown that this type of conformers indeed can be the most energetically beneficial. The uncertainty in equilibrium geometry led to exclusion some of the hydroxide from the final version of the database due to computational problems at the coupled clusters stage.

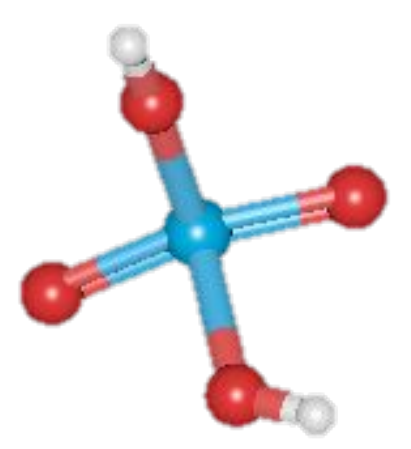

$\mathrm{UO}_{2}(\mathrm{OH})_{2}$

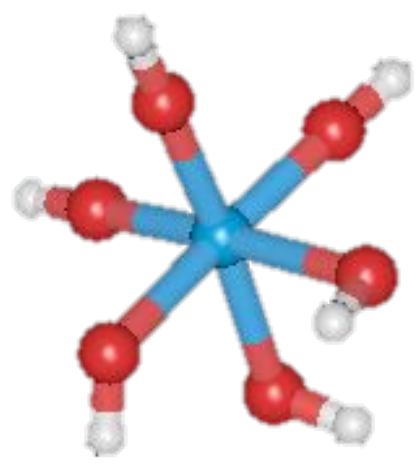

$\mathrm{Np}(\mathrm{OH})_{6}$, not featured in this article

Fig. S2 Asymmetric hydroxide structures. 


\section{Deviation of the angle in oxications}

In actinide compounds with oxication such as uranyl salts, we observed angle between oxygens and uranium atom less than 180 degrees. This effect has already been discussed by other scientific groups ${ }^{3,4}$.

\section{Complete basis set error}

The basis set error seemed to be a significant problem for the coupled-clusters calculations. Unfortunately, for a similar task (the presence of actinide atoms, the need of relativistic corrections, number of exponents per orbital and the software has a limit on a number of basis functions) there are no suitable basis sets for a complete basis set extrapolation except Jorge basis family ${ }^{5}$. We've tried to test it on several simple reactions with uranium atom:

$U+2 F_{2}->U F_{4}$

$\mathrm{UF}_{4}+\mathrm{F}_{2} \rightarrow \mathrm{UF}_{6}$

$\mathrm{U}(\mathrm{OH})_{4}+4 \mathrm{HF}->\mathrm{UF}_{4}+4 \mathrm{H}_{2} \mathrm{O}$

$\mathrm{UF}_{6}+2 \mathrm{H}_{2} \mathrm{O}->\mathrm{UO}_{2} \mathrm{~F}_{2}+4 \mathrm{HF}$

It is seen (Table S1) that the energy of uranium-containing molecules computed with double-zeta basis is less than the energy of the same molecule computed with triple-zeta basis. While the behavior on the other compounds is opposite. Also, some calculations have refused to converge, so we were not able to construct any basis extrapolation technique.

Table S1. Computation results at the level of coupled clusters theory with Jorge basis family.

\begin{tabular}{|c|c|c|c|}
\hline Compound & Basis set name & $\mathrm{E}$, hartree & $E_{\text {extrap, }}$ hartree \\
\hline \multirow{2}{*}{$\mathrm{HF}$} & jorge-DZP-DKH & $-100,3007784$ & \multirow{2}{*}{$-100,49$} \\
\hline & jorge-TZP-DKH & $-100,4354155$ & \\
\hline \multirow{2}{*}{$\mathrm{F}_{2}$} & jorge-DZP-DKH & $-199,2400179$ & \multirow{2}{*}{$-199,61$} \\
\hline & jorge-TZP-DKH & $-199,4981921$ & \\
\hline \multirow{2}{*}{ U } & jorge-DZP-DKH & $-27944,91761$ & \multirow{2}{*}{$-27941,00$} \\
\hline & jorge-TZP-DKH & $-27942,15946$ & \\
\hline \multirow{2}{*}{$\mathrm{UF}_{4}$} & jorge-DZP-DKH & corrupted & \multirow{2}{*}{ N/A } \\
\hline & jorge-TZP-DKH & corrupted & \\
\hline \multirow{2}{*}{$\mathrm{UF}_{6}$} & jorge-DZP-DKH & $-28543,80529$ & \multirow{2}{*}{$-28541,04$} \\
\hline & jorge-TZP-DKH & $-28541,85476$ & \\
\hline \multirow{2}{*}{$\mathrm{UO}_{2} \mathrm{~F}_{2}$} & jorge-DZP-DKH & $-28295,06707$ & \multirow{2}{*}{$-28291,87$} \\
\hline & jorge-TZP-DKH & $-28292,81803$ & \\
\hline \multirow{2}{*}{$\mathrm{U}(\mathrm{OH})_{4}$} & jorge-DZP-DKH & corrupted & \multirow{2}{*}{ N/A } \\
\hline & jorge-TZP-DKH & $-28245,83184$ & \\
\hline
\end{tabular}

\section{Popular quantum chemistry methods vs. $\operatorname{CCSD}(\mathrm{T})$ approach}

As we mentioned in the article, we have only a few examples of well-studied reactions to test the method used in the article. And the majority of quantum-chemical calculations of actinide compounds are usually performed with simple DFT approached. Here we used a set of simple reactions (Table S2) to compare the results obtained by popular DFT approaches with the perturbation theory, the CCSD(T) method, and as its local variant (DLPNO). The figure S3 shows the unsigned deviations of reaction energies calculated by different methods. The coupled cluster calculations were taken as a baseline. 
Table S2. Selected reactions for methods comparison.

\begin{tabular}{|c|c|}
\hline$\#$ & Reaction \\
\hline$I$ & ${U F_{4}+4 \mathrm{H}_{2} \mathrm{O}->\mathrm{U}(\mathrm{OH})_{4}+4 \mathrm{HF}} \mathrm{UCl}_{6}+3 \mathrm{~F}_{2}>\mathrm{UF}_{6}+3 \mathrm{Cl}_{2}$ \\
\hline $\mathrm{II}$ & $\mathrm{UF}_{4}+\mathrm{F}_{2}>\mathrm{UF}_{6}$ \\
\hline $\mathrm{II}$ & $\mathrm{U}+3 \mathrm{Cl}_{2}>\mathrm{UCl}_{6}$ \\
\hline $\mathrm{V}$ & $\mathrm{U}+3 \mathrm{~F}_{2}>\mathrm{UF}_{6}$ \\
\hline $\mathrm{VI}$ & $\mathrm{U}+2 \mathrm{~F}_{2}>\mathrm{UF}_{4}$ \\
\hline $\mathrm{VII}$ & $\mathrm{UF}_{6}+2 \mathrm{H}_{2} \mathrm{O}>\mathrm{UO}_{2} \mathrm{~F}_{2}+4 \mathrm{HF}$ \\
\hline
\end{tabular}

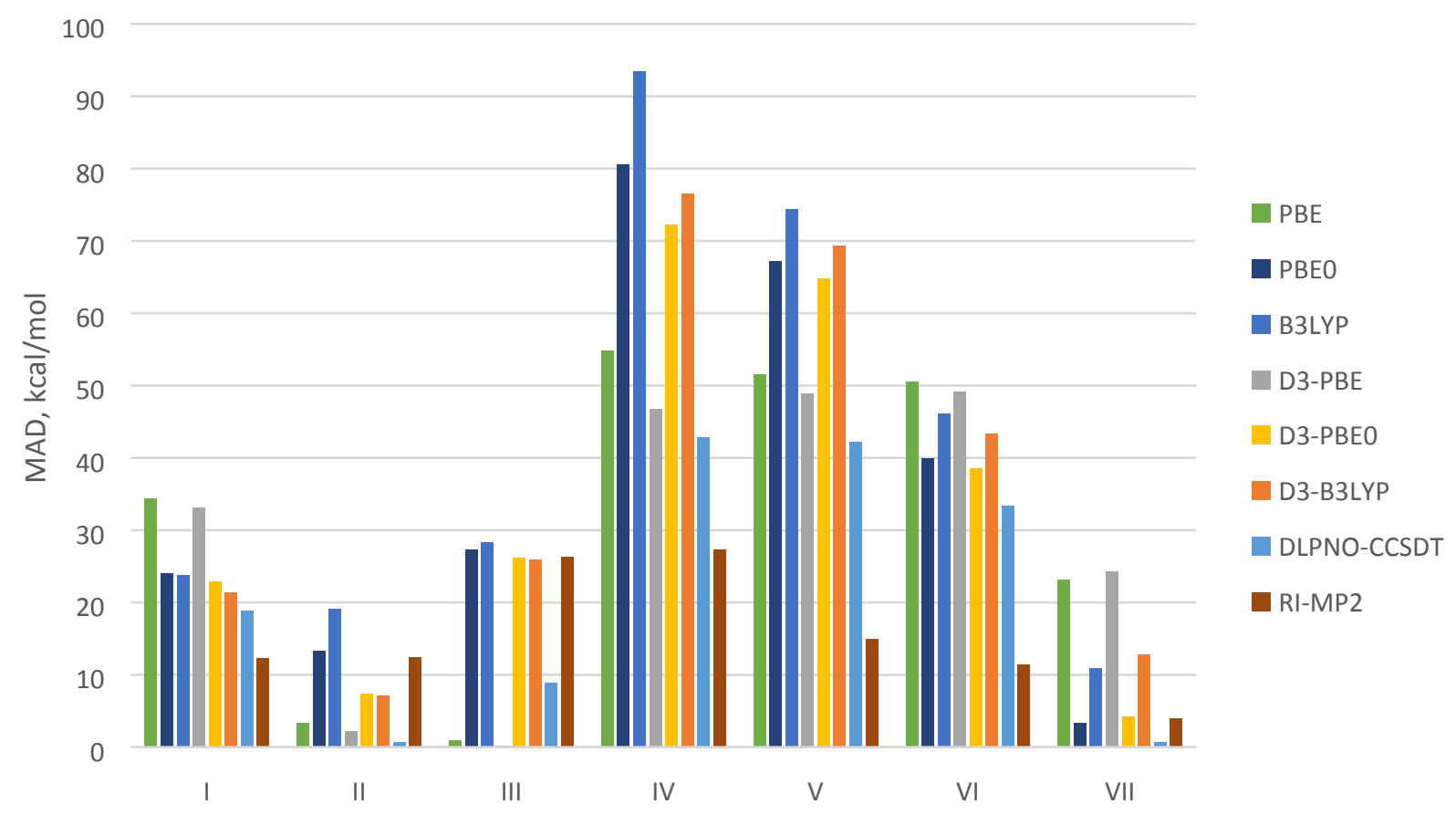

Fig. S3 MAD of popular DFT methods for selected reactions.

Thus, popular DFT methods demonstrate a huge deviation from the reference CCSD $(T)$ values and they are not suitable for such type of compounds. It is also interesting, that domain-based local pair natural orbital CC method (DLPNO-CCSDT) that usually gives adequate energetic results for transition elements' chemistry, for actinide compounds shows poor results. Thus, the best results were obtained from RI-MP2 method. Though, the topic needs further testing and development.

\section{The benchmarking}

The following table shows the computational time for $\operatorname{CCSD}(T)$ calculations. It is interesting to notice, it is hard to see any correlation with the number of basis set functions in the system. Though the reason may be in a relatively small size of the dataset. 


\begin{tabular}{|c|c|c|c|c|c|c|c|c|c|c|}
\hline $\begin{array}{l}\text { Compound } \\
\text { name }\end{array}$ & Is UHF? & $\begin{array}{c}\text { Number of } \\
\text { basis } \\
\text { functions }\end{array}$ & $\begin{array}{l}\text { Number of } \\
\text { contracted } \\
\text { basis } \\
\text { functions }\end{array}$ & $\begin{array}{l}\text { Full time, } \\
\text { sec }\end{array}$ & $\begin{array}{l}\text { SCF time, } \\
\text { sec }\end{array}$ & $\begin{array}{c}\text { MDCl time, } \\
\text { sec }\end{array}$ & $\begin{array}{c}\text { Number of } \\
\text { threads }\end{array}$ & $\begin{array}{l}\text { Number of } \\
\text { SCF } \\
\text { iterations }\end{array}$ & $\begin{array}{l}\text { Number of } \\
\qquad \mathrm{MDCl} \\
\text { iterations }\end{array}$ & $\begin{array}{c}\text { CPU * time } \\
\text { (UHF } \\
\text { normalized) }\end{array}$ \\
\hline $\mathrm{UCl}_{6}$ & False & 631 & 381 & 3360 & 31 & 3313 & 20 & 8 & 12 & 67206 \\
\hline $\mathrm{UF}_{4}$ & True & 437 & 287 & 4674 & 40 & 4613 & 20 & 24 & 13 & 186952 \\
\hline $\mathrm{UF}_{5}$ & True & 483 & 319 & 482302 & 1128 & 481173 & 1 & 32 & 60 & 964604 \\
\hline $\mathrm{UF}_{6}$ & False & 529 & 351 & 2986 & 56 & 2926 & 20 & 22 & 11 & 59720 \\
\hline $\mathrm{UO}_{2}\left(\mathrm{NO}_{3}\right)_{2}$ & False & 713 & 479 & 22388 & 128 & 22241 & 20 & 26 & 11 & 447756 \\
\hline $\mathrm{UO}_{2}(\mathrm{OH})_{2}$ & False & 453 & 299 & 4435 & 403 & 4028 & 5 & 30 & 11 & 22174 \\
\hline $\mathrm{UO}_{2} \mathrm{C}_{2} \mathrm{O}_{4}$ & False & 621 & 415 & 27045 & 536 & 26504 & 5 & 25 & 12 & 135227 \\
\hline $\mathrm{UO}_{2} \mathrm{Cl}_{2}$ & False & 471 & 297 & 22006 & 1921 & 20082 & 1 & 25 & 12 & 22006 \\
\hline $\mathrm{UO}_{2} \mathrm{CO}_{3}$ & False & 529 & 351 & 12587 & 648 & 11935 & 5 & 30 & 13 & 62936 \\
\hline $\mathrm{UO}_{2} \mathrm{~F}_{2}$ & False & 437 & 287 & 23440 & 32 & 23399 & 20 & 1 & 11 & 468794 \\
\hline $\mathrm{UO}_{2} \mathrm{SO}_{4}$ & False & 592 & 388 & 22065 & 737 & 21323 & 4 & 30 & 13 & 88259 \\
\hline $\mathrm{Ac}(\mathrm{HCOO})_{3}$ & False & 691 & 465 & 30202 & 137 & 30057 & 10 & 8 & 8 & 302021 \\
\hline $\mathrm{Ac}(\mathrm{NO} 3)_{3}$ & False & 805 & 543 & 219482 & 56 & 219418 & 4 & 1 & 10 & 877929 \\
\hline $\mathrm{Ac}(\mathrm{OH})_{3}$ & False & 415 & 273 & 6173 & 465 & 5707 & 1 & 19 & 8 & 6173 \\
\hline $\mathrm{AcCl}_{3}$ & False & 442 & 270 & 1326 & 196 & 1127 & 5 & 24 & 8 & 6632 \\
\hline $\mathrm{AcF}_{3}$ & False & 391 & 255 & 4494 & 365 & 4127 & 1 & 19 & 8 & 4494 \\
\hline $\mathrm{Am}(\mathrm{OH})_{3}$ & True & 415 & 273 & 46422 & 1482 & 44939 & 1 & 56 & 15 & 92844 \\
\hline $\mathrm{AmF}_{3}$ & True & 391 & 255 & 32608 & 1043 & 31560 & 1 & 53 & 13 & 65215 \\
\hline $\mathrm{Bk}(\mathrm{OH})_{3}$ & True & 415 & 273 & 67569 & 4874 & 62692 & 2 & 184 & 27 & 270277 \\
\hline $\mathrm{Cm}(\mathrm{OH})_{3}$ & True & 415 & 273 & 99032 & 1781 & 97249 & 1 & 23 & 12 & 198064 \\
\hline $\mathrm{CmF}_{3}$ & True & 391 & 255 & 45426 & 747 & 44678 & 1 & 23 & 11 & 90853 \\
\hline $\mathrm{Np}(\mathrm{OH})_{3}$ & True & 415 & 273 & 61153 & 1966 & 59184 & 1 & 74 & 27 & 122306 \\
\hline $\mathrm{Np}(\mathrm{OH})_{4}$ & True & 469 & 311 & 190676 & 2380 & 188294 & 1 & 66 & 50 & 381351 \\
\hline $\mathrm{Np}\left(\mathrm{SO}_{4}\right)_{2}$ & True & 747 & 489 & 385723 & 87 & 385627 & 20 & 14 & 32 & 15428920 \\
\hline $\mathrm{NpF}_{4}$ & True & 437 & 287 & 193326 & 5134 & 188190 & 1 & 201 & 65 & 386651 \\
\hline $\mathrm{NpF}_{6}$ & True & 529 & 351 & 63961 & 27 & 63925 & 20 & 9 & 12 & 2558420 \\
\hline $\mathrm{NpO}_{2} \mathrm{~F}$ & True & 391 & 255 & 11920 & 405 & 11511 & 5 & 48 & 14 & 119200 \\
\hline $\mathrm{NpO}_{2} \mathrm{HCOO}$ & True & 491 & 325 & 84662 & 1863 & 82795 & 4 & 105 & 46 & 677293 \\
\hline
\end{tabular}




\begin{tabular}{|c|c|c|c|c|c|c|c|c|c|c|}
\hline $\mathrm{NpO}_{2} \mathrm{OH}$ & True & 399 & 261 & 16767 & 1907 & 14856 & 5 & 260 & 26 & 167668 \\
\hline $\mathrm{PaF}_{4}$ & True & 437 & 287 & 8939 & 60 & 8875 & 21 & 38 & 28 & 375437 \\
\hline $\mathrm{PaOCl}_{3}$ & False & 488 & 302 & 3721 & 337 & 3380 & 4 & 26 & 10 & 14883 \\
\hline $\mathrm{PaOF}_{3}$ & False & 437 & 287 & 684 & 37 & 630 & 20 & 22 & 10 & 13681 \\
\hline $\mathrm{Pu}(\mathrm{OH})_{3}$ & True & 415 & 273 & 84102 & 2407 & 81693 & 1 & 82 & 39 & 168204 \\
\hline $\mathrm{Pu}(\mathrm{OH})_{4}$ & True & 469 & 311 & 111143 & 2032 & 109110 & 1 & 59 & 19 & 222287 \\
\hline $\mathrm{PuCl}_{6}$ & False & 631 & 381 & 19232 & 1160 & 18065 & 5 & 50 & 14 & 96160 \\
\hline $\mathrm{PuF}_{3}$ & True & 391 & 255 & 21691 & 1008 & 20635 & 21 & 172 & 134 & 911001 \\
\hline $\mathrm{PuF}_{4}$ & True & 437 & 287 & 9997 & 68 & 9913 & 20 & 41 & 27 & 399896 \\
\hline $\mathrm{PuF}_{6}$ & False & 529 & 351 & 56405 & 1389 & 55013 & 1 & 29 & 13 & 56405 \\
\hline $\mathrm{PuO}_{2}(\mathrm{OH})_{2}$ & True & 453 & 299 & 75442 & 1916 & 73524 & 2 & 72 & 16 & 301769 \\
\hline $\mathrm{PuO}_{2} \mathrm{C}_{2} \mathrm{O}_{4}$ & True & 621 & 415 & 81078 & 463 & 80609 & 20 & 79 & 15 & 3243137 \\
\hline $\mathrm{PuO}_{2} \mathrm{Cl}_{2}$ & True & 471 & 297 & 59747 & 1025 & 58717 & 5 & 81 & 49 & 597470 \\
\hline $\mathrm{PuO}_{2} \mathrm{CO}_{3}$ & True & 529 & 351 & 221954 & 3115 & 218835 & 4 & 185 & 65 & 1775630 \\
\hline $\mathrm{PuO}_{2} \mathrm{SO}_{4}$ & True & 592 & 388 & 458804 & 2262 & 456536 & 4 & 104 & 77 & 3670429 \\
\hline $\operatorname{Th}\left(\mathrm{C}_{2} \mathrm{O}_{4}\right)_{2}$ & False & 805 & 543 & 60018 & 305 & 59704 & 20 & 28 & 11 & 1200364 \\
\hline $\mathrm{Th}\left(\mathrm{CO}_{3}\right)_{2}$ & False & 621 & 415 & 22861 & 463 & 22392 & 5 & 21 & 10 & 114304 \\
\hline $\mathrm{Th}\left(\mathrm{NO}_{3}\right)_{4}$ & False & 989 & 671 & 1015281 & 2293 & 1012974 & 4 & 29 & 10 & 4061124 \\
\hline $\mathrm{Th}(\mathrm{OH})_{4}$ & False & 469 & 311 & 826 & 36 & 771 & 21 & 19 & 7 & 17347 \\
\hline $\mathrm{Th}\left(\mathrm{SO}_{4}\right)_{2}$ & False & 747 & 489 & 118127 & 6179 & 111939 & 5 & 196 & 10 & 590635 \\
\hline $\mathrm{ThCl}_{4}$ & False & 505 & 307 & 675 & 19 & 652 & 20 & 7 & 8 & 13498 \\
\hline $\mathrm{ThF}_{4}$ & False & 437 & 287 & 1541 & 28 & 1510 & 15 & 7 & 8 & 23117 \\
\hline $\mathrm{U}(\mathrm{OH})_{4}$ & False & 469 & 311 & 1752 & 143 & 1605 & 21 & 86 & 33 & 36796 \\
\hline $\mathrm{UCl}_{4}$ & True & 505 & 307 & 134457 & 1455 & 133000 & 1 & 42 & 40 & 268914 \\
\hline $\mathrm{UCl}_{5}$ & True & 568 & 344 & 174050 & 89 & 173953 & 1 & 1 & 18 & 348099 \\
\hline
\end{tabular}




\section{References}

(1) Wang, Q.; Pitzer, R. M. Structure and Spectra of UO2F2 and Its Hydrated Species. J. Phys. Chem. A 2001, 105 (36), 8370-8375. https://doi.org/10.1021/jp004009z.

(2) Añez, R.; Alejos, P.; Sierraalta, A. HAl(OH)2 Molecular Structures and Reaction Paths. PostHartree-Fock, DFT Calculations and Infrared Spectroscopic. Comput. Theor. Chem. 2015, 1060, 31-35. https://doi.org/10.1016/j.comptc.2015.02.008.

(3) Hayton, T. W. Understanding the Origins of Oyl-U-Oyl Bending in the Uranyl (UO2 2+) Ion. Dalt. Trans. 2018, 47 (4), 1003-1009. https://doi.org/10.1039/C7DT04123C.

(4) Conn, G. K. T.; Wu, C. K. An Investigation of the Raman and Infra-Red Spectra of Certain Uranyl Salts, with Conclusions on the Structure of the Uranyl Group. Trans. Faraday Soc. 1938, 34 (0), 1483. https://doi.org/10.1039/tf9383401483.

(5) Jorge, F. E.; Canal Neto, A.; Camiletti, G. G.; Machado, S. F. Contracted Gaussian Basis Sets for Douglas-Kroll-Hess Calculations: Estimating Scalar Relativistic Effects of Some Atomic and Molecular Properties. J. Chem. Phys. 2009, 130 (6), 064108. https://doi.org/10.1063/1.3072360. 